\title{
Inhibitors of the p38 cell signaling pathway as antiviral compounds against Junín virus
}

\author{
Jesús Emanuel Brunetti ${ }^{1,2,3} \cdot$ Verónica Mara Quintana ${ }^{1,2,4} \cdot$ Luis Alberto Scolaro ${ }^{1,2} \cdot$ Viviana Castilla $^{1}$ (1)
}

Received: 25 October 2021 / Accepted: 4 January 2022 / Published online: 8 February 2022

(c) The Author(s), under exclusive licence to Springer-Verlag GmbH Austria, part of Springer Nature 2022

\begin{abstract}
In the present study, we analyzed the modulation of p38 cell signaling by Junín virus (JUNV) and evaluated the antiviral activity of p38 inhibitors against JUNV. While JUNV induced a progressive activation of p38 throughout the infection in Vero cells, a partial downregulation of p38 phosphorylation was observed in HEK293 and HeLa cells. The compounds SB203580 and SB202190, which are selective inhibitors of p38, significantly reduced viral protein expression and viral yield in the cell lines examined, indicating that the p38 signaling pathway might be a promising antiviral target against JUNV infection.
\end{abstract}

Members of the genus Mammarenavirus (family Arenaviridae) are enveloped single-stranded RNA viruses that cause persistent infections in their natural reservoirs. Some members of the genus can cause hemorrhagic fever in humans, such as the Old World mammarenavirus (OWM) Lassa virus (LASV), which is endemic in Africa, and the New World mammarenavirus (NWM) Junín virus (JUNV), the etiological agent of Argentine hemorrhagic fever (AHF) [1].

JUNV establishes a persistent infection in the cricetid Calomys musculinus, which is mainly distributed in the central region of Argentina, and humans become infected through inhalation of animal body fluids or excretions through agriculture practices. Vaccination with the live attenuated Candid \#1 vaccine has markedly reduced the incidence of AHF. However, Candid \#1 is not recommended

Handling Editor: Tim Skern.

Viviana Castilla

viviana@qb.fcen.uba.ar

1 Laboratorio de Virología, Departamento de Química Biológica, Facultad de Ciencias Exactas y Naturales, Universidad de Buenos Aires (UBA), 1428 Buenos Aires, Argentina

2 IQUIBICEN, Consejo Nacional de Investigaciones Científicas y Técnicas (CONICET)-UBA, Ciudad Universitaria, 1428 Buenos Aires, Argentina

3 Present Address: Virus Immunology Group, Bernhard Nocht Institute for Tropical Medicine, Hamburg, Germany

4 Present Address: Instituto de Investigaciones Biotecnológicas, Universidad Nacional de San Martín, 1650 Buenos Aires, Argentina for children and pregnant women. Administration of convalescent plasma is the approved treatment for AHF patients, but it is effective only when it is initiated early in infection. Novel antiviral therapies are needed for patients with delayed diagnosis and to prevent shortages of immune plasma [2].

Viruses can manipulate mitogen-activated protein kinase (MAPK)-dependent cell signaling pathways to ensure their efficient replication. Several DNA and RNA viruses modulate the p38 MAPK pathway, which is involved in many cellular processes, including the inflammatory response, apoptosis, autophagy, and senescence [3-5]. The four p38 isoforms ( $\alpha, \beta, \gamma$, and $\delta$ ) have some redundant functions but also display differences in the substrates they activate [3]. Specific inhibitors of the $\alpha$ and $\beta$ isoforms of $\mathrm{p} 38$ have been developed, including the compounds SB203580, SB202190, and losmapimod. SB203580 and SB202190 compete with ATP for binding to the active site of p38. SB202190 inhibits both p38 phosphorylation and activity, whereas SB203580 inhibits p38 catalytic activity without preventing its phosphorylation [6]. Inhibition of p38 signaling impairs in vitro replication of RNA viruses, including respiratory viruses $[4,7,8]$, enterovirus 71 [9], rotaviruses (RVs) [10], flaviviruses [5, 11-13], Chikungunya virus [14], and severe acute respiratory syndrome coronavirus 2 (SARS-CoV-2) $[5,15]$.

Little is known about the participation of p38 signaling in arenavirus replication. p38 phosphorylation has been shown to be unaffected in mouse fibroblasts infected with the OWM lymphocytic choriomeningitis virus (LCMV) [16]. However, activation of $\mathrm{p} 38$ has been observed in guinea pig macrophages and murine monocytes infected with Pichindé virus, an NWM [17]. Interestingly, the p38 inhibitor 


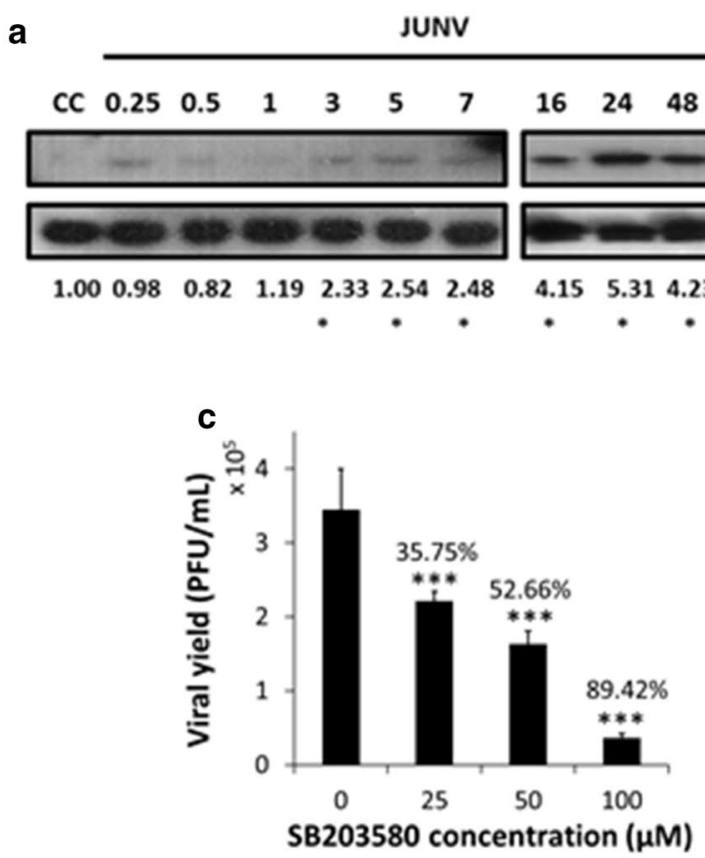

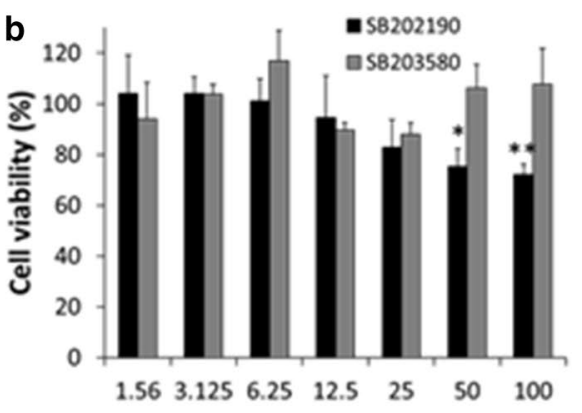

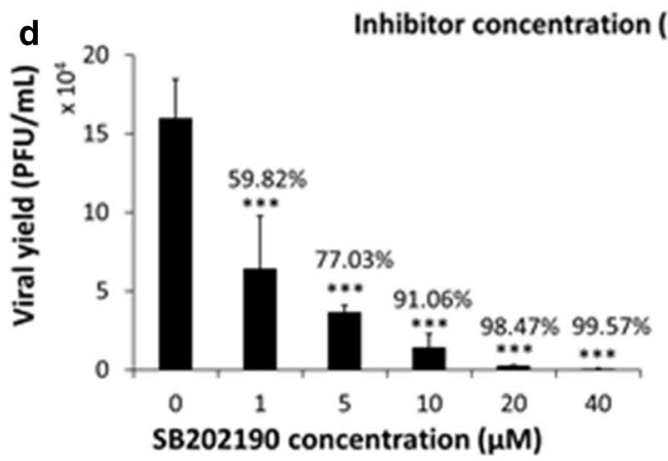

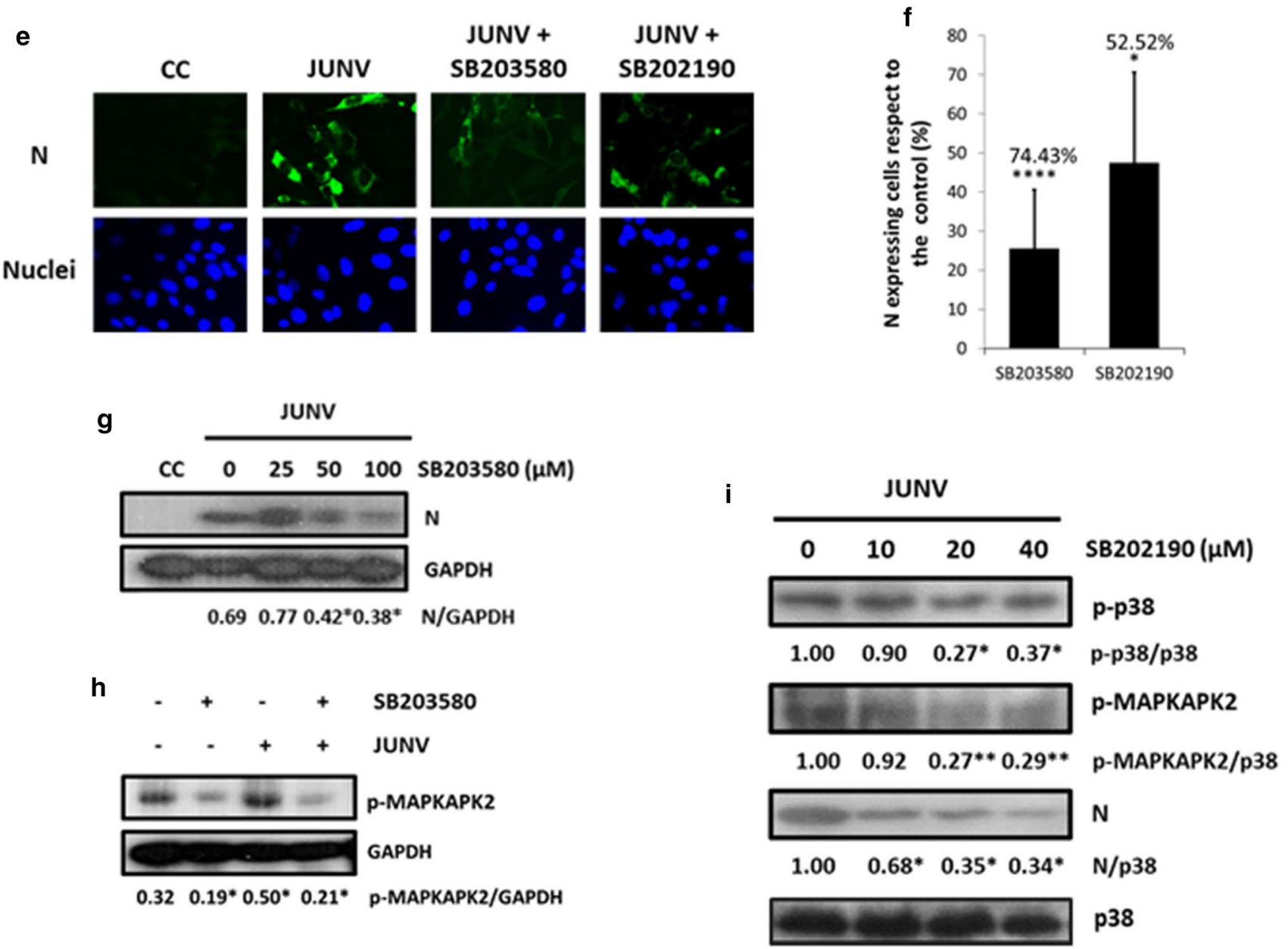


4Fig. 1 Inhibition of p38 signaling impairs JUNV replication in Vero cells. (a) JUNV-infected (MOI =1) Vero cells were harvested at different time points p.i., and the levels of p-p38 and p38 were assessed by WB. The ratio of p-p38 to p38 with respect to the mock-infected culture (cc) is indicated. AN: cells treated with anisomycin, a p38 activator. (b) Vero cell cultures were incubated with different concentrations of SB203580 or SB202190, and after $24 \mathrm{~h}$, cell viability was evaluated using an MTT assay. (c and d) JUNV-infected Vero cells $(\mathrm{MOI}=1)$ were treated with different concentrations of SB203580 or SB202190 for $24 \mathrm{~h}$, and viral titers were determined by plaque assay. The percentage of inhibition of viral yield with respect to untreated cultures is indicated above the bars. (e) JUNV-infected cultures $(\mathrm{MOI}=1)$ were treated with SB203580 $(100 \mu \mathrm{M})$ or SB202190 $(40 \mu \mathrm{M})$, and at $24 \mathrm{~h}$ p.i., $\mathrm{N}$ protein expression was detected by IF, cell nuclei were stained with Hoechst, and (f) the percentage of $\mathrm{N}$-expressing cells was determined by counting 20 fields selected at random. The numbers above the bars indicate the percentage of reduction with respect to untreated infected cultures. (g) JUNVinfected Vero cells $(\mathrm{MOI}=1)$ were treated with different concentrations of SB203580, and at $24 \mathrm{~h}$ p.i., $\mathrm{N}$ and GAPDH expression was assessed by WB; the ratio N/GAPDH is indicated. (h) Mock-infected or JUNV-infected Vero cells were treated with SB203580 $(100 \mu \mathrm{M})$, and at $24 \mathrm{~h}$ p.i., p-MAPKAPK2 and GAPDH expression was assessed by $\mathrm{WB}$; the ratio p-MAPKAPK2/GAPDH is indicated. (i) JUNVinfected Vero cells $(\mathrm{MOI}=1)$ were treated with different concentrations of SB202190, and at 24 h p.i., p-p38, N, and p38, expression was detected by WB. The ratios p-p38/p38, p-MAPKAPK2/p38, and $\mathrm{N} / \mathrm{p} 38$ with respect to untreated infected cultures are indicated. In a-d and $\mathrm{f}-\mathrm{i}$, data are mean values \pm standard deviation (SD) from three independent experiments. ANOVA (Dunnett post-hoc test) was used in statistical analysis: *, $p<0.05$; **, $p<0.01$; ***, $p<0.001$; ****, $p<0.0001$

losmapimod has been shown to impair LASV entry into the host cell, but the antiviral activity of this compound is not achieved through inhibition of the $\mathrm{p} 38$ pathway [18]. In previous studies, we demonstrated the involvement of ERK1/2 MAPK in JUNV replication [19, 20]. Here, we investigated the effect of JUNV infection on p38 signaling in monkey and human cell lines and the antiviral effect of inhibitors of the p38 pathway against JUNV.

To determine whether JUNV infection modulates p38 signaling, Vero cells (monkey kidney fibroblasts, ATCC CCL-81) were infected with JUNV (strain XJCl3) at a multiplicity of infection (MOI) of 1 and incubated in MEM (Gibco) without serum. At different times postinfection (p.i.), p38 phosphorylation was examined by Western blot (WB) as described previously [12]. The activation of p38 was initially detected at $3 \mathrm{~h}$ p.i., whereas higher phosphop38 (p-p38) levels were evident from 16 to $72 \mathrm{~h}$ p.i. (Fig. 1a). To investigate the importance of p38 activation in JUNV infection, we first examined the effect of the p38 inhibitors SB203580 and SB202190 (Promega) on the viability of Vero cells using the MTT (3-(4,5-dimethylthiazol-2yl)2,5-diphenyl tetrazolium bromide) (Sigma-Aldrich) method [20] (Fig. 1b). Then, JUNV-infected Vero cells were treated with non-cytotoxic concentrations of each compound. At $24 \mathrm{~h}$ p.i., virus yields were determined by plaque assay, whereas viral $\mathrm{N}$ protein expression was analyzed either by immunofluorescence (IF) or WB assay as described previously [20]. Both inhibitors reduced JUNV yields in a dosedependent manner (Fig. 1c and d). Moreover, treatment with the highest concentration of SB203580 $(100 \mu \mathrm{M})$ or SB202190 $(40 \mu \mathrm{M})$ caused a significant reduction in the number of cells expressing the $\mathrm{N}$ protein (Fig. 1e and $\mathrm{f}$ ). A dose-dependent inhibition of $\mathrm{N}$ protein expression was confirmed by WB in cells treated with SB203580 (Fig. 1g) or SB202190 (Fig. 1i). The inhibition of p38 activation by SB203580 was confirmed by analyzing the phosphorylation level of MAPK-activated protein kinase 2 (MAPKAPK2), a downstream effector of p38 kinase (Fig. 1h), using rabbit anti-p-MAPKAPK2 (\#3007 Cell Signaling Technology) as the primary antibody for WB. Treatment with SB202190 (20 and $40 \mu \mathrm{M}$ ) caused a significant reduction in p-p38 and p-MAPKAPK2, as shown in Fig. 1i.

Then, a time course assay was conducted to examine the phosphorylation state of p38 in HEK-293 cells (human embryonic kidney, ATCC CRL-1573) infected with JUNV. In contrast to the results obtained with Vero cells, JUNVinfected HEK-293 cells exhibited lower levels of p-p38 than uninfected cultures at $24 \mathrm{~h}$ p.i. A gradual increase in p-p38 levels was observed between 24 and 72 h p.i. (Fig. 2a). The percentage of N-protein-expressing cells in HEK-293 cultures also gradually increased throughout the infection (Figs. 2b and c), suggesting that the spread of the infection promotes $\mathrm{p} 38$ activation. At the same time points, Vero cells exhibited higher percentages of $\mathrm{N}$-expressing cells than HEK-293 cells (Fig. 2c). However, the data did not differ significantly between the two cell types, so it is possible that specific cellular factors influence p38 activation. The highest non-cytotoxic concentration of SB202190 (50 $\mu \mathrm{M})$ assayed (Fig. 2d) exerted a non-significant reduction in the p-p38 level in HEK-293 cells (Fig. 2f). However, the viral yield and $\mathrm{N}$ protein expression were markedly reduced (Fig. 2e and $\mathrm{f}$ ).

In line with the results obtained in HEK-293 cells, JUNV infection of HeLa cells (human epithelial cervix cells, ATCC CRM-CCL-2) also caused a decrease in p-p38 levels in comparison with mock-infected cultures at $24 \mathrm{~h}$ p.i. (Fig. 3a). However, inhibition of basal levels of p38 activation (Fig. 3a) in cells treated with non-cytotoxic concentrations of SB202190 (Fig. 3b) resulted in a striking decrease in $\mathrm{N}$ protein expression (Fig. 3a) and virus yield (Fig. 3c).

Therefore, JUNV-mediated modulation of p38 signaling is dependent on the cell line assessed. In accordance with our results in Vero cells, a gradual increase in p-p38 levels throughout the infection has been reported for picornaviruses in dendritic [9] and epithelial human cells [7], SARS-CoV in Vero E6 cells [21], and SARS-CoV-2 in human-lung-derived cells [15]. However, JUNV induced a slight downregulation of p38 phosphorylation in HEK-293 and HeLa cells at 24 h p.i. Unlike Vero cells, HEK-293 and HeLa cells are 

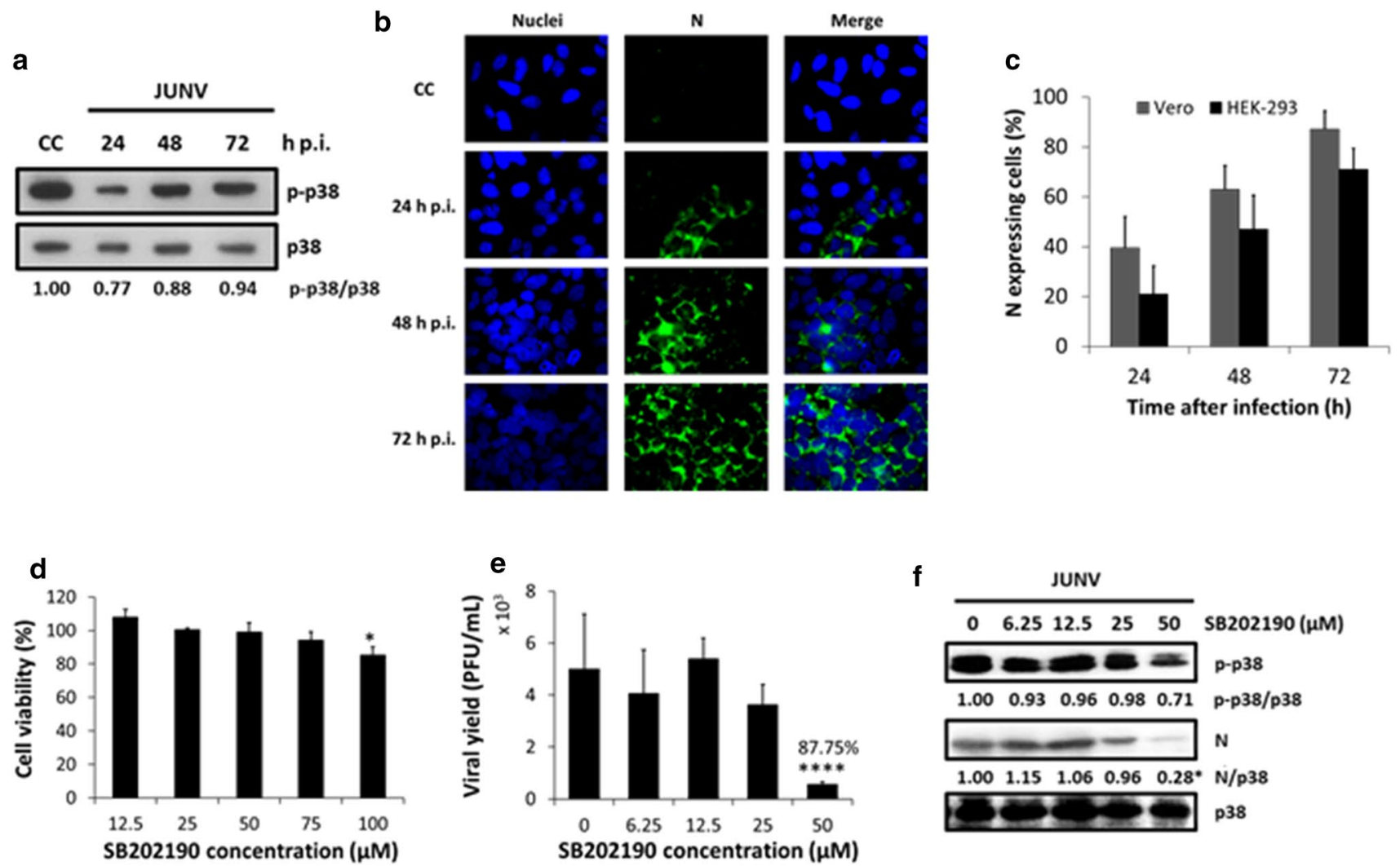

Fig. 2 SB202190 inhibits JUNV replication in HEK-293 cells. (a) JUNV-infected (MOI =1) HEK-293 cells were lysed at different time points after infection, and levels of p-p38 and p38 were assessed by WB. The ratio p-p38/p38 with respect to mock-infected cultures (cc) is indicated. (b) $\mathrm{N}$ protein expression detected by IF in JUNV-infected $(\mathrm{MOI}=1)$ HEK-293 cells at different times p.i. Nuclei were stained with Hoescht, and (c) the percentage of $\mathrm{N}$-expressing cells in Vero or HEK-293 cells was determined by counting 20 fields selected at random. (d) HEK-293 cells were treated with different concentrations of SB202190, and after $24 \mathrm{~h}$, cell viability was evaluated using an MTT assay. (e) JUNV-infected (MOI=1) HEK-293 cells were treated with different concentrations of SB202190, and at $24 \mathrm{~h}$ p.i., viral titers were determined by plaque assay. The percentage of inhibition of viral yield with respect to untreated cultures is indicated above the bars (f) JUNV-infected (MOI=1) HEK-293 cells were incubated with different concentrations of SB202190, and at $24 \mathrm{~h}$ p.i., levels of p-p38, N, and p38 were assessed by WB; the ratios p-p38/p38 and $\mathrm{N} / \mathrm{p} 38$ with respect to untreated infected cultures are indicated. In a and $\mathrm{c}-\mathrm{f}$, the data are mean values $\pm \mathrm{SD}$ from three independent experiments. ANOVA (Dunnett post-hoc test) was used in statistical analysis: *, $p<0.05$; **, $p<0.01 ; * * * *, p<0.0001$

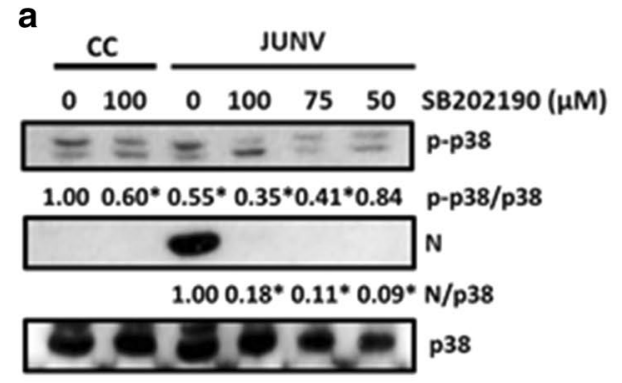

Fig. 3 SB202190 inhibits JUNV replication in HeLa cells. (a) Mockinfected (cc) or JUNV-infected (MOI=1) HeLa cells were treated with SB202190, and at $24 \mathrm{~h}$ p.i., the levels of p-p38, N, and p38 were assessed by WB. The ratio p-p38/p38 with respect to uninfected cells and the ratio $\mathrm{N} / \mathrm{p} 38$ with respect to untreated infected cultures are indicated. (b) HeLa cell cultures were treated with different concentrations of SB202190, and after 24 h, cell viability was assessed by
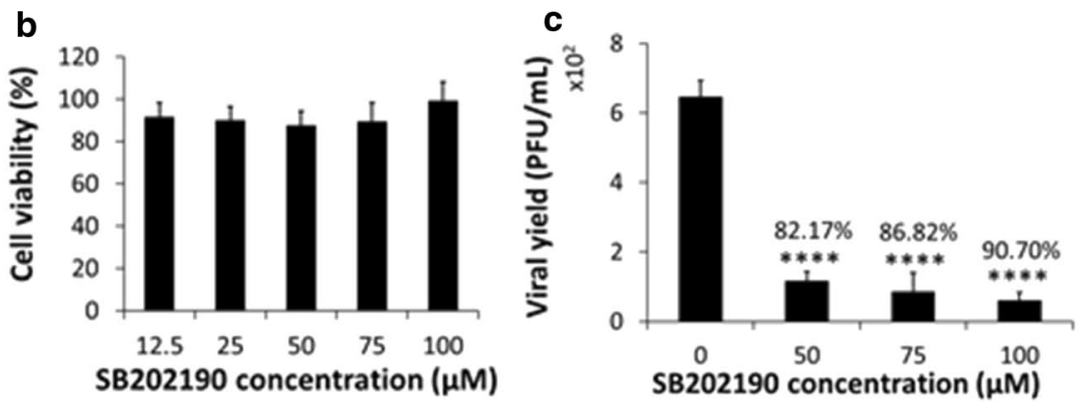

MTT assay. (c) JUNV-infected $(\mathrm{MOI}=1) \mathrm{HeLa}$ cells were treated with different concentrations of SB202190, and at $24 \mathrm{~h}$ p.i., viral titers were determined by plaque assay. The numbers above the bars indicate the percentage of inhibition of viral yield with respect to untreated infected cultures. In a-c, data are mean values from three independent experiments \pm SD. ANOVA (Dunnett post-hoc test) was used in statistical analysis: *, $p<0.05 ; * * * *, p<0.0001$ 
interferon (IFN) type 1 producers [22-24]. It has been shown that IFN exhibits only a moderate inhibitory effect on JUNV replication [24], probably due to the ability of the viral proteins $\mathrm{N}$ and $\mathrm{Z}$ to counteract the antiviral activity of IFN [25]. Therefore, it is possible that, soon after JUNV infection, IFN induction in human cells partially restrains or delays the synthesis of viral factors responsible for $\mathrm{p} 38$ activation and that, as the infection progresses, there is a gradual increase in p-p38 levels. Furthermore, downregulation of p38 phosphorylation associated with the presence of IFN $\alpha$ has been described in Huh 7.5.1 cells infected with hepatitis $C$ virus [26]. Nevertheless, we cannot rule out the possibility that other cell-specific factors influence JUNV modulation of p38. Indeed, the OWM LCMV does not induce changes in p38 phosphorylation in mouse fibroblasts [16] but reduces p38 activation in mouse dendritic cells [27]. Moreover, the kinetics of p38 activation by influenza A virus, respiratory syncytial virus, and adenovirus vary according to the cell type [7], while RV induces p38 activation in Caco-2 cells but not in HT-29 cells [10].

A substantial decrease in JUNV replication was evident in Vero, HEK-293, and HeLa cells following inhibition of p38 by chemical compounds. In spite of the fact that noncytotoxic concentrations of p38 inhibitors only partially suppressed p38 activation, this incomplete inhibition was apparently enough to significantly impair viral protein expression and virus production, indicating that functional p38 signaling plays an important role in JUNV replication. The non-cytotoxic concentrations of p38 inhibitors tested did not reduce ERK MAPK phosphorylation (data not shown). However, off-target effects of the compounds cannot be ruled out entirely. By contrast, blocking the p38 pathway using chemical inhibitors or small interfering RNAs did not impair in vitro OWM LASV replication [18].

Cell signaling pathways represent an attractive target for new antiviral strategies with a low risk of the development of viral resistance. This is the first demonstration of modulation of p38 signaling due to JUNV infection. Severe infections with pathogenic NWM correlate with strong induction of pro-inflammatory cytokines [25], and p38 signaling is a key regulator of the inflammatory response. In addition, MAPKs have been implicated in the establishment of persistent viral infections [21]. Therefore, the role of p38 in JUNV replication, pathogenesis, and persistence in its rodent reservoir deserves further investigation. Although it remains to be investigated if SB203580 and SB202190 show in vivo efficacy against JUNV, several highly selective p38 inhibitors that are active at low nanomolar concentrations have been developed for the treatment of different diseases [8, 28]. Some of these compounds have good safety profiles and are therefore potential candidates for the treatment of NWM infections.
Acknowledgements This work was supported by a grant from the Universidad de Buenos Aires (20020190100211BA). LAS is a member of the Research Career from Consejo Nacional de Investigaciones Científicas y Técnicas (CONICET).

\section{Declarations}

Conflict of interest The authors have no relevant financial or non-financial interests to disclose.

\section{References}

1. Brisse ME, Ly H (2019) Hemorrhagic fever-causing arenaviruses: lethal pathogens and potent immune suppressors. Front Immunol 10:372. https://doi.org/10.3389/fimmu.2019.00372

2. Enría DA, Briggiler AM, Sánchez Z (2008) Treatment of Argentine hemorrhagic fever. Antiviral Res 78(1):132-139. https://doi. org/10.1016/j.antiviral.2007.10.010

3. Martínez-Limón A, Joaquin M, Caballero M, Posas F, de Nadal E (2020) The p38 pathway: from biology to cancer therapy. Int J Mol Sci 21(1913):1-18. https://doi.org/10.3390/ijms21061913

4. Meineke R, Rimmelzwaan GF, Elbahesh H (2019) Influenza virus infections and cellular kinases. Viruses 11(2):171. https://doi.org/ 10.3390/v11020171

5. Cheng Y, Sun F, Wang L et al (2020) Virus-induced p38 MAPK activation facilitates viral infection. Theranostics 10(26):1222312240. https://doi.org/10.7150/thno.50992

6. Bühler S, Laufer SA (2014) p38 MAPK inhibitors: a patent review (2012-2013). Expert Opin Ther Patents 24(5):535-554. https:// doi.org/10.1517/13543776.2014.894977

7. Marchant D, Singhera GK, Utokaparch S et al (2010) Toll-Like receptor 4-mediated activation of $\mathrm{p} 38$ mitogen-activated protein kinase is a determinant of respiratory virus entry and tropism. J Virol 84(21):11359-11373. https://doi.org/10.1128/JVI.00804-10

8. Choi M-S, Heo J, Yi C-M et al (2016) A novel p38 mitogen activated protein kinase (MAPK) specific inhibitor suppresses respiratory syncytial virus and influenza A virus replication by inhibiting virus-induced p38 MAPK activation. Biochem Biophys Res Commun 477:311-316. https://doi.org/10.1016/j.bbrc.2016.06.111

9. Peng H, Shi M, Zhang L et al (2014) Activation of JNK1/2 and p38 MAPK signaling pathways promotes enterovirus 71 infection in immature dendritic cells. BMC Microbiol 14(1):1-9. https:// doi.org/10.1186/1471-2180-14-147

10. Holloway G, Coulson BS (2006) Rotavirus activates JNK and p38 signaling pathways in intestinal cells, leading to AP-1-driven transcriptional responses and enhanced virus replication. J Virol 80(21):10624-10633. https://doi.org/10.1128/JVI.00390-06

11. Cheng F, Ramos da Silva S, Huang I-C, Jung JU, Gao S-J (2018) Suppression of Zika virus infection and replication in endothelial cells and astrocytes by PKA inhibitor PKI 14-22. J Virol 92(4):117. https://doi.org/10.1128/JVI.02019-17

12. Quintana VM, Selisko B, Brunetti JE et al (2020) Antiviral activity of the natural alkaloid anisomycin against dengue and Zika viruses. Antiviral Res 176:104749. https://doi.org/10.1016/j.antiv iral.2020.104749

13. Sugasti-Salazar M, Llamas-González YY, Campos D, GonzálezSantamaría J (2021) Inhibition of p38 mitogen-activated protein kinase impairs Mayaro virus replication in human dermal fibroblasts and HeLa cells. Viruses 13(6):1156. https://doi.org/10. 3390/v13061156

14. Mudaliar P, Pradeep P, Abraham R, Sreekumar E (2021) Targeting cap-dependent translation to inhibit Chikungunya virus replication: selectivity of p38 MAPK inhibitors to virus-infected cells 
due to autophagy-mediated down regulation of phospho-ERK. J Gen Virol 102(7). https://doi.org/10.1099/jgv.0.001629

15. Bouhaddou M, Memon D, Meyer B et al (2020) The global phosphorylation landscape of SARS-CoV-2 infection. Cell 182(3):685712.e19. https://doi.org/10.1016/j.cell.2020.06.034

16. Renukaradhya GJ, Webb TJR, Khan MA et al (2005) Virusinduced inhibition of CD1d1-mediated antigen presentation: reciprocal regulation by p38 and ERK. J Immunol 175:43014308. https://doi.org/10.4049/jimmunol.175.7.4301

17. Bowick GC, Fennewald SM, Scott EP et al (2007) Identification of differentially activated cell-signaling networks associated with pichinde virus pathogenesis by using systems kinomics. J Virol 81(4):1923-1933. https://doi.org/10.1128/JVI.02199-06

18. Zhang $X$, Yan F, Tang $\mathrm{K}$ et al (2019) Identification of a clinical compound losmapimod that blocks Lassa virus entry. Antiviral Res 167:68-77. https://doi.org/10.1016/j.antiviral.2019.03.014

19. Rodríguez ME, Brunetti JE, Wachsman MB, Scolaro LA, Castilla V (2014) Raf/MEK/ERK pathway activation is required for Junín virus replication. J Gen Virol 95:799-805. https://doi.org/ 10.1099/vir.0.061242-0

20. Brunetti JE, Foscaldi S, Quintana VM et al (2018) Role of the ERK1/2 signaling pathway in the replication of Junín and Tacaribe viruses. Viruses 10(4):1-15. https://doi.org/10.3390/v1004 0199

21. Mizutani T, Fukushi S, Ishii K et al (2006) Mechanisms of establishment of persistent SARS-CoV-infected cells. Biochem Biophys Res Commun 347(1):261-265. https://doi.org/10.1016/j. bbrc.2006.06.086

22. Ank N, West H, Bartholdy $\mathrm{C}$ et al (2006) Lambda interferon (IFN$\lambda$ ), a type III IFN, is induced by viruses and IFNs and displays potent antiviral activity against select virus infections in vivo. $\mathrm{J}$ Virol 80(9):4501-4509. https://doi.org/10.1128/JVI.80.9.45014509.2006
23. Spiegel M, Weber F (2006) Inhibition of cytokine gene expression and induction of chemokine genes in non-lymphatic cells infected with SARS coronavirus. Virol J 3(17). https://doi.org/10.1186/ 1743-422X-3-17

24. Huang C, Kolokoltsova OA, Yun NE et al (2012) Junín virus infection activates the type I interferon pathway in a RIG-Idependent manner. PLoS Negl Trop Dis 6(5):e1659. https://doi. org/10.1371/journal.pntd.0001659

25. Mantlo E, Paessler S, Huang C (2019) Differential immune responses to hemorrhagic fever-causing arenaviruses. Vaccines (Basel) 7(4):138. https://doi.org/10.3390/vaccines7040138

26. He SF, Wang W, Ren H, Zhao LJ, Qi ZT (2013) Interferon alpha and ribavirin collaboratively regulate $\mathrm{p} 38$ mitogen-activated protein kinase signaling in hepatoma cells. Cytokine 61(3):801-807. https://doi.org/10.1016/j.cyto.2013.01.007

27. Che Mat NF, Siddiqui S, Mehta D et al (2018) Lymphocytic choriomeningitis virus infection of dendritic cells interferes with TLR-induced IL-12/IL-23 cytokine production in an IL-10 independent manner. Cytokine 108:105-114. https://doi.org/10.1016/j. cyto.2018.03.017

28. Haller V, Nahidino P, Forster M, Laufer SA (2020) An updated patent review of p38 MAP kinase inhibitors (2014-2019). Expert Opin Ther Patents 30(6):453-466. https://doi.org/10.1080/13543 776.2020 .1749263

Publisher's Note Springer Nature remains neutral with regard to jurisdictional claims in published maps and institutional affiliations. 